\title{
The horizon-ratio relation as information for relative size in pictures
}

\author{
SHEENA ROGERS \\ University of Wisconsin, Madison, Wisconsin
}

\begin{abstract}
The horizon-ratio relation was found to be an effective source of information for relative size in pictures under some conditions: when the difference in image size of depicted "same real size" objects was not too great (Experiment 1), and when the horizon line was not too high or too low in the picture (Experiment 2). The latter finding seemed to be linked to the observers' identification of the horizontal line as the horizon (and not as the edge of a finite surface). In addition, individual patterns of response were remarkably systematic even in the absence of a horizon, or any other pictorial information, (Experiment 3). It is suggested that in this case observers imposed a horizon on the picture on which to base their relative size judgments, possibly based on the observer's own eye level or on the content of the picture. It is concluded that although the horizon-ratio relation provides the same kind of information as that available in the optic arrays from real scenes, pictorial information requires the satisfaction of additional constraints in order to be fully effective.
\end{abstract}

Questions concerning the perception of spatial layout in pictures have traditionally been framed in terms of "pictorial cues" (e.g., height in the picture, relative size, interposition). Research has tended to focus on the development of sensitivity to these cues in infants and young children and, to a lesser extent, among adults from non-Western cultures (see, e.g., Benson \& Yonas, 1973; Granrud, Yonas, \& Opland, 1985; Ireson \& McGurk, 1985; Jahoda \& McGurk, 1974; Olson, 1975; Olson \& Boswell, 1976; Wilcox \& Teghtsoonian, 1971; Yonas \& Hagen, 1973). Less attention has been paid to how adults, within our own culture, successfully perceive depicted spatial relations.

New questions were raised, however, by the alternative approach to visual perception proposed by J. J. Gibson (1966, 1979). Gibson argued that perception does not require the construction of space from local and punctate cues but rather the direct detection of invariant optical structures that are informative about properties of the environment. Gibson also extended this theory to account for the perception of pictures. He claimed that there is an intrinsic structural relation between pictures and the real scenes they represent; that there are informative structures in pictures; and that these structures are of the same sort as those found in the changing optic array from a real scene (J. J. Gibson, 1971, 1979).

Gibson's followers have been keen to maintain the case for the structural equivalence of pictures and scenes (see, e.g., the contributors to Hagen, 1980). So far, however, little has been provided by way of example of invariant structure in the changing optic array that might also constitute a possible pictorial "invariant." Little, that is, until Sedg-

Correspondence may be sent to S. Rogers, Department of Psychology, University of Wisconsin, 1202 W. Johnson St., Madison, WI 53706 (e-mail: sheenar@macc.wisc.edu). wick (1973) identified the horizon as the basis of a series of invariant optical relations between the horizon and the projection in the array of all objects on the ground plane. He subsequently considered how these relations might be defined in terms of the projective geometry of pictorial perspective (Sedgwick, 1980).

The horizon, defined as the infinitely distant limit of a ground plane, is not a feature of the environment but of the optic array, and its position in the array is always at eye level. Hence, the length of an object from its base on the ground to the point where it intersects the horizon is always the same as the height of the point of observation above the ground. Knowledge of the height of the point of observation would allow accurate estimates to be made of the height and width of all objects standing on the ground plane, which are also vertical and parallel to the picture plane. In a picture, the height of the point of observation may not be known. If more than one object is present in the scene, however, their relative sizes are fully specified by the horizon-ratio relation. Figure 1 illustrates an example. Object $\mathrm{A}$ is intersected at its midpoint by the horizon, and it is therefore twice the height of the point of observation. A second object, $B$, is intersected at one third its height and is thus three times the height of the point of observation. Object $B$ is therefore one and one half times as tall as A. Objects of the same height, $B$ and $C$ in the example figure, are intersected in the same proportion by the horizon, regardless of the distance between the objects and the observer. ${ }^{1}$ The horizon ratio, then, concerns two distances measured on the picture plane (or measured in terms of visual angle if the optic array rather than the picture itself is considered): Let $S$ be the distance between the base of the object and its top, and let $\mathrm{H}$ be the distance between the base of the object and the horizon. Notice that the ratio $\mathrm{S} / \mathrm{H}$ (the horizon ratio) is identical for Objects $\mathrm{B}$ and $\mathrm{C}$, indicating their equality. 


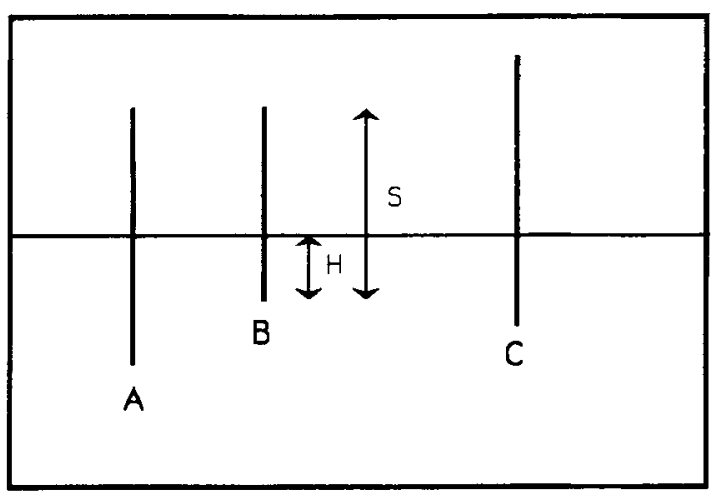

Figure 1. The horizon ratio is given by $\mathrm{S} / \mathrm{H}$, where $\mathrm{S}$ is the distance from the base of the object to its top and $H$ is the distance from the base of the object to the horizon. Object $A$ is twice the height of the point of observation (horizon ratio $=2$ ); Objects $B$ and $C$ are three times the height of the point of observation (horizon ratio $=3$ ). Objects $B$ and $C$ are each one and a half times as tall as Object $A$.

Sedgwick's $(1973,1980)$ analysis of the horizon relations shows that the horizon is a basis of a potentially powerful source of information for spatial relations in pictures. Although Sedgwick has shown that the information is available, as yet there is little evidence that it is actually used by picture viewers, and this essential task remains. In two studies of perceived tree size, Bingham (1993a, 1993b) indirectly investigated the effectiveness of the horizonratio relation. His observers judged the absolute size of trees and the size of cylinders on the ground surface, and he reported that the horizon-ratio relation did not account for performance at the task. However, Bingham's cylinders were of the same image size and may not have fully been integrated into the pictorial space. In this case, they could appear to be affixed to the picture surface, and the horizon-ratio relation would not apply. (The role of eyeheight information as a scaling factor in the perception of real scenes has also been considered recently and there is some evidence of its usefulness. See, e.g., Mark, 1987; Warren, 1984; Warren \& Whang, 1987.) The question of the effectiveness of available information (i.e., its utilization by observers) is crucial to Gibson's case. Specifically, the immediate task is to determine whether the horizonratio relation is effective in the perception of depicted spatial relations.

The results of a preliminary exploratory study strongly suggested that the horizon-ratio relation can indeed provide an effective basis for relative size judgments in pictures (Rogers \& Costall, 1983). In this earlier study, very simple stimulus pictures were constructed. Most included a horizontal line drawn across the picture surface; all featured a vertical line representing a tall object standing on the ground plane and a small cross that marked the base of a second object. The subjects' task was to draw in the second line so that it appeared to be of the same "real size" as the given standard (i.e., so that it could represent a similar object of the same size, but at a different distance).
Some of the stimulus pictures included additional pictorial features such as linear perspective or a texture gradient, and in some, the horizontal line depicted was not the true horizon (the vanishing line of the ground plane). Individuals were tested in one condition only and most subjects' responses to the task were remarkably accurate. The horizonratio relation, unlike traditional cues such as height in the picture and relative size, specifies relative height precisely, and accurate responses should therefore be expected if the observers' judgments are based on this structure. It seems likely, then, that many participants in this preliminary study were indeed making use of the available horizon-based information. ${ }^{2}$

Three experiments are reported in the present paper which extend this main finding and examine two possible challenges to the notion that horizon relations are a reliable source of information in pictorial size perception. An accurate response in the earlier study entailed the drawing of a farther and therefore pictorially smaller comparison. The range of possible responses was limited, so perhaps there was less opportunity for participants to go badly wrong. Experiment 1 examined the extent to which subjects could maintain accuracy in a similar adjustment task over multiple trials with greater variety in the positioning of picture elements and thus greater variety in the length of the response required relative to the given standard in order to produce a same real size match. Experiments 2 and 3 each addressed an aspect of the results of the earlier study that suggested that the relationship between pictorial structure and perception is not as straightforward as Gibson's theory would suppose. First, the location of the horizon in the picture plane seemed to be an important variable. More accurate relative size judgments were made when the horizon was in the upper half of the picture. This finding is difficult to interpret in Gibson's terms and therefore required further study (Experiment 2). Second, subjects' responses to the drawing task in pictures where no horizon was given (and where one could not be recovered from other pictorial structures) were nevertheless quite consistent. Sedgwick (1980) had suggested that an implicit horizon might be recovered in a perspective picture (and Rogers and Costall's [1983] results supported this suggestion). However, he also proposed that the horizon might be recovered when not even implicitly defined, perhaps by registering the observer's own eye level on the picture plane (Sedgwick, 1980, p. 86). This imaginary horizon, he argued, could also support the horizon relations and allow estimates of relative size to be made. Although this notion could fit within Gibson's definition of pictorial information, it does not easily predict the consistency that was found between subjects in the earlier study (Rogers \& Costall, 1983). This curious finding was the subject of further investigation in the present Experiment 3.

All three experiments investigated possible developmental differences in sensitivity to horizon-based information among adults within the same culture. E. J. Gibson and J. J. Gibson (1955) suggested that learning, in the form of the education of attention to available sources of information, may take place in perception. In the present ex- 
periments, the performance of people with considerable experience of representational pictures (graduate students of graphic design and illustration from the Royal College of Art, London) was therefore compared with that of a control group comprising graduate students in disciplines other than art and design.

\section{EXPERIMENT 1}

The relative pictorial size of two objects with the same real size is given precisely by the horizon-ratio relation. If the pictorial size of a standard object and its position relative to the horizon are fixed, there will be a linear decrease in the length of the (same real size) comparison object as its base is elevated in the picture plane toward the horizon. At the horizon, the comparison object will be infinitely small. Experiment 1 investigated the precision with which observers' responses track this relationship, in an adjustment task similar to that in Rogers and Costall's (1983) study, but with a repeated measures design. For ease of presentation and subsequent analysis, the displays were generated and the experiment was controlled by computer. The line length was increased and decreased from the keyboard arrow keys, giving more precise control over the length of the response line than was possible with the pencil-and-paper technique used by Rogers and Costall.

The elevation of the starting point was varied from the bottom of the picture to the horizon, so that the depicted distance of the comparison line changed from trial to trial. When the starting point is lower in the picture than the base of the standard, the appropriate comparison needs to be longer than the standard to produce a "same real size" match, a situation not examined previously. If the horizonratio relation is effective and is based on the given horizon, the starting-point manipulation should produce responses that closely follow the predicted negative linear relationship. If not, a number of outcomes are possible. Response lengths could decrease linearly, but too quickly or too slowly; the relationship could be systematic but nonlinear; or responses could be unsystematic.

An additional variable was introduced: A standard line of fixed length was drawn at one of two elevations so that it was either intersected by the horizon (and so depicted an object taller than eye height) or was entirely below the horizon (depicting an object smaller than eye height). The horizon relations apply to all objects standing on the ground plane, and it is not a requirement that they be intersected by the horizon. Figure 2 shows how the the relative size of objects that do not extend above eye level can be determined by the horizon-ratio relation. It is conceivable that the ratio judgment underlying performance of the task is more easily made in one condition or the other (perhaps when the standard is actually intersected by the horizon). A comparison of performance in these two conditions permits a test of this idea.

\section{Method}

Subjects. There were 9 "artists" and 5 control subjects from the groups described above.

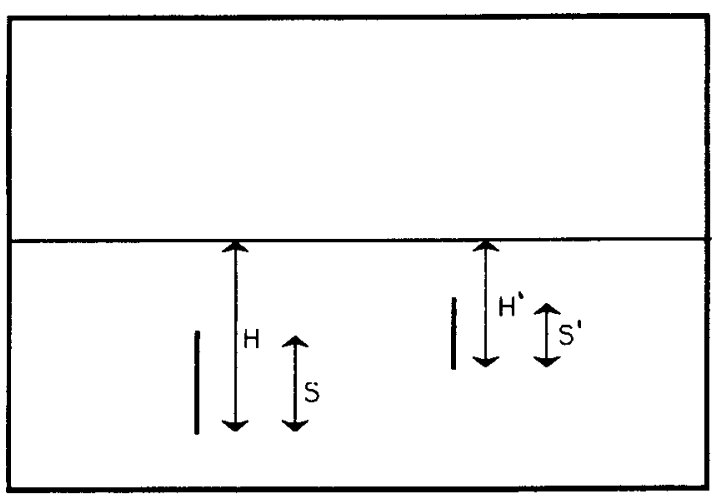

Figure 2. The horizon ratio does not require objects to be intersected by the horizon. Both objects are below eye height; but $\mathrm{S} / \mathrm{H}=\mathrm{S}^{\prime} / \mathrm{H}^{\prime}=0.5$, so they have the same real height.

Design and pictorial displays. The displays were computer generated, and the dimensions of the displays were originally set in numbers of pixels. These have been converted to millimeters throughout the report (there are approximately 5.25 pixels per millimeter). The picture border was $230 \mathrm{~mm}$ (horizontal) $\times 195 \mathrm{~mm}$ (vertical). The horizon was fixed just above the halfway point at $114 \mathrm{~mm}$. The standard line was $28.5 \mathrm{~mm}$ long, and it was positioned at one of two elevations: with its base either $38 \mathrm{~mm}$ or $95 \mathrm{~mm}$ from the bottom border. In the first case, the standard was entirely below the horizon, and in the second, the standard was intersected by the horizon at two thirds its height. In both cases, on any trial, the standard line appeared at either one third the picture width from the left or from the right border. This made a total of four positions available for random selection by the computer on any trial.

The starting point for the response was a one pixel dot. On any trial it appeared somewhere between $2.85 \mathrm{~mm}$ from the bottom border and $2.85 \mathrm{~mm}$ from the horizon, in $2.85-\mathrm{mm}$ steps, for a total of 39 possible elevations. Again the horizontal placement of the starting point varied randomly from trial to trial, appearing at one quarter of the picture width from the left, from the right, or half way. In this way, each of the 39 trials presented a novel display to the subject.

Thus there was one between-subjects factor, subject group (with two levels, artists and controls); and two within-subjects experimental factors, the elevation of the starting point for the response (with 39 levels) and the elevation of the standard line (with two levels, intersected [high] and not intersected [low]). The last two factors were not crossed, in order to avoid excessive numbers of trials and repetitive responding by observers. Instead, each elevation of the starting point was sampled once only in any run. Random selection of the position of the standard on each trial ensured that each subject made about half of the 39 adjustments with a low standard and half with the intersected standard. (There were no grounds for expecting an interaction between these two factors.)

Apparatus. A BBC Model B microcomputer was used to generate the displays, control the experiment, and collect the data. Displays were presented on a Microvitec Cub high-resolution 14-in. graphics monitor.

Procedure. Subjects were tested individually. The display monitor was on a table in front of the subject at a comfortable height. Free, unrestricted viewing of the displays was allowed because the experiment was intended to test the effectiveness of a potential pictorial invariant under normal viewing conditions. The following instructions were given:

Imagine that the picture represents a real scene. It shows a flat, horizontal surface. Resting on the surface and perpendicular to it is a stick or a pole. I would like you to draw another line, starting at this dot, so that it appears to be the same real height as the standard, but farther away. 
No indication was given of the identity or function of the horizon line. Subjects were shown how to increase and decrease the length of the response with the arrow keys on the computer keyboard at the beginning of the first trial. Pressing another key ended each trial and started the next. At the end of the session, subjects were asked to explain how they had performed the task and whether they could identify the horizontal line.

\section{Results and Discussion}

The horizon-ratio rule predicts a negative linear relationship between the elevation of the starting point and the length of the response required to produce a "same real size" match. The slope and intercept of this function depend on the particular configuration of horizon and standard used in the picture. Figure 3 plots the mean obtained response length as a function of the elevation of the starting point, for artists and control subjects, in the low-standard condition (panel a) and in the high-standard condition (panel $b$ ). The dotted line indicates the matches predicted from the horizon-ratio relation.

Regression analyses were performed on the data of each subject separately to obtain a coefficient of determination $\left(r^{2}\right)$, slope and $y$-intercept in both low- and highstandard conditions. In each case, response length was re-

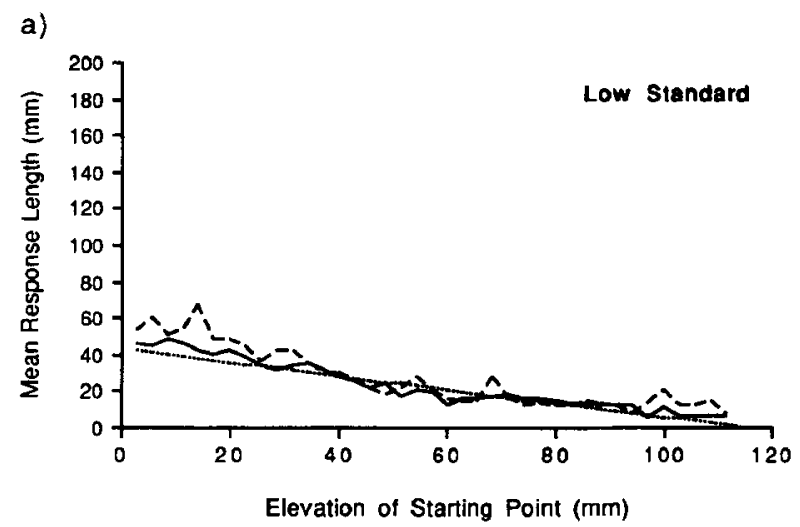

b)

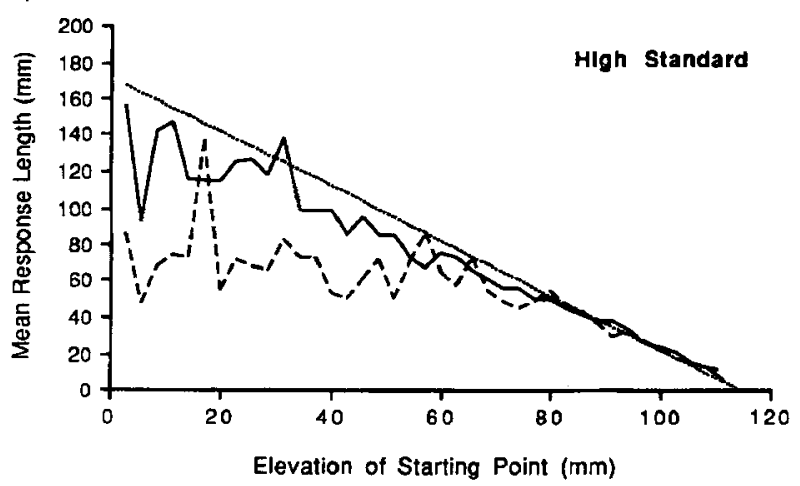

Figure 3. Experiment 1: Mean obtained response length for artists and control subjects as a function of the elevation of the starting point (a) when the standard is low in the picture and (b) when the standard is high (intersected). The solid line indicates the artists' responses, and the broken line indicates those of the controls. The straight dotted line indicates the response length predicted by the horizon-ratio relation. gressed on the predicted value of the response length for each elevation of the starting point. Table 1 gives the mean slope, $r^{2}$, and $y$-intercept for the artists and controls for both positions of the standard, with standard errors.

The regression line of response length on prediction will have slope 1 and intercept 0 if perfect performance is obtained. Response curves were strongly linear and systematic as predicted, for both subject groups in both conditions (see Table 1). In the low-standard condition, the mean slope of the curves was not significantly different from 1 for both the artists and the controls [artists' mean slope, $1.07, t(8)=1.143$; controls' mean slope, 1.128 , $t(4)=0.528$, n.s.]. In the high-standard condition, the artists' mean slope, .775, was significantly lower than 1 $[t(8)=3.285, p<.05]$, as was the controls' [mean slope, $.4 ; t(4)=5.576, p<.01]$. Thus performance in the lowstandard condition, when the standard was below the horizon, closely matched the predictions from the horizonratio relation (see Figure $3 a$ ). When the standard was high and intersected by the horizon, performance was poorer. Inspection of Figure $3 \mathrm{~b}$ reveals that, in this condition, responses were accurate in both subject groups until conditions required subjects to draw a very tall response- that is, one at least three times the length of the standard. In this case, both groups produced shorter than expected responses and two of the control subjects, in particular, seemed reluctant to draw such a long line.

The regression analyses indicate, however, that the horizon-ratio relation accounts significantly for performance in both conditions (low and high standard) for all except 1 control subject whose responses were markedly discrepant in the high-standard condition (significant individual $F$ ratios ranged from 40.471 to 684.095 in the low-standard condition, and from 41.909 to 1274.177 in the high-standard condition; $p<.0001$ in all cases). Individual slopes for the 13 accurate subjects were significantly non-zero at $p<.0001$ and a very large amount of the total variance in each subject's responses can be accounted for by the horizon-ratio relation predictions (overall mean $r^{2}=.878$, for 13 subjects).

A small increment in $r^{2}$ was obtained in testing for a quadratic component in each subject's function (low-standard mean $I=0.056$, high-standard mean $I=0.071$ ). Error variance was very low for most subjects, so $I$ was significant for all but 3 subjects in each standard condition $(p<.05)$. However, we can rule out the alternative hypothesis that the function is curved because the quadratic component is very small in relation to the large linear component (low-standard mean $r^{2}=0.859$, high-standard mean $r^{2}=0.874$, excluding the deviant control subject). The small quadratic component in the high-standard condition is apparent in Figure $3 \mathrm{~b}$ as underestimations of response length at the lower elevations of the starting point.

The deviant subject, in the control group, responded reasonably accurately in the low-standard condition $\left[r^{2}=\right.$ $.547 ; F(1,18)=21.724, p<.0002]$, but her responses were erratic and did not show the systematic linear decrease in length with increasing elevation of the starting point in the high-standard condition $\left[r^{2}=.061 ; F(1,17)=1.01\right.$, n.s. $]$. 
Table 1

Means of Individual Regression Analyses for Experiment 1

(Horizon Present) for Artists and Controls in High- (Intersected)

and Low- (Not Intersected) Standard Conditions

\begin{tabular}{lcccccc}
\hline \multicolumn{7}{c}{ and Low- } \\
Condition & $r^{2}$ & $S E$ & Slope & $S E$ & $y$-Intercept & $S E$ \\
\hline & & & Artists & & & \\
Low & .909 & .032 & 1.07 & .061 & 0.389 & 1.465 \\
High & .896 & .028 & .775 & .068 & 8.233 & 2.704 \\
& & & Controls & & & \\
Low & .767 & .06 & 1.128 & .242 & 1.624 & 4.348 \\
High & .672 & .158 & .4 & .108 & 21.82 & 4.153 \\
\hline
\end{tabular}

Two two-factor analyses of variance (ANOVAs) with subject group as a between-subjects factor and standard position as a within-subjects factor were computed by using the individual slopes and $r^{2} \mathrm{~s}$. The ANOVA of the slopes revealed no overall difference in the accuracy of performance between artist and control subjects [artists' mean slope $=.923$, controls' mean slope $=.764 ; F(1,12)=$ 1.487 , n.s.]. Slopes were significantly different between stimulus conditions, however. That is, overall accuracy was good in the low-standard condition (mean slope = $1.091)$ but significantly poorer in the high-standard condition [mean slope $=.641, F(1,1)=28.838, p<.0002$ ] This difference, of course, cannot be explained by the horizonratio rule. The tendency was for subjects to draw the response too short in the high-standard condition. In addition, the especially poor performance by control subjects in the high-standard condition led to a significant interaction between subject group and stimulus condition $[F(1,12)=5.163, p<.05]$.

The $r^{2}$ coefficient is highest when all of a subject's responses lie very close to the regression line, indicating very systematic behavior and precise adjustment from trial to trial ( $r^{2}$ s over .9 were achieved by 11 subjects). The ANOVA of the $r^{2} \mathrm{~s}$ revealed a close-to-significant difference on this measure between artists and controls [artists' mean $r^{2}=$ .903 , controls' mean $r^{2}=.72 ; F(1,12)=4.668, p=$ .0516]. This difference is entirely due to the single deviant control subject, however, and it completely disappeared when the data were reanalyzed without her [controls' mean $r^{2}=.824 ; F(1,11)=3.13$, n.s.]. There was no effect of standard position on the $r^{2}$ measure [high-standard mean $r^{2}=.816$, low-standard mean $r^{2}=.859 ; F(1,1)=1.563$, n.s.] and no interaction $[F(1,12)=.9$, n.s.].

In summary, the presence of a horizon in otherwise informationless pictures makes possible extremely accurate estimates of the relative sizes of depicted objects, within some limits. Extreme differences in the pictorial size of depicted "same real size" objects were not tolerated by observers in this experiment, who, instead, underestimated the response size when very large responses were required. There is a suggestion that observers with more experience of pictures (the "artists" here) are somewhat more tolerant of this strong foreshortening than are ordinary observers.

\section{EXPERIMENT 2}

The horizon-ratio relation appears to be able to support judgments of relative size in pictures. If this pictorial invariant provides information in J. J. Gibson's sense, then accurate judgments should be made wherever in the picture plane the horizon is located. In Experiment 2, the horizon was positioned at one of five elevations in simple line drawings on paper. Each drawing included a vertical standard line and a small cross as a starting point for a drawn comparison. These two features remained at a constant position in each picture, so that the length of the drawn response should increase with each increase in the elevation of the horizon in order to match the horizon ratio of the standard. If differences in accuracy were observed with changes in the position of the horizon, the notion of pictorial invariants and their role in picture perception would require qualification.

\section{Method}

Subjects. There were 41 "artists" and 35 control subjects from the groups described above. Different individuals took part in this experiment. The subjects were randomly assigned to conditions giving 11 artists and 5 controls in Condition A (see below for conditions); 9 artists and 7 controls in B; 5 artists and 9 controls in $\mathrm{C} ; 9$ artists and 6 controls in D; and 7 artists and 8 controls in $\mathrm{E}$.

Pictorial displays. A set of four simple line drawings was constructed on thin card, each $210 \times 150 \mathrm{~mm}$. A vertical standard line $100 \mathrm{~mm}$ in length was drawn on the left, $25 \mathrm{~mm}$ from the bottom of the picture. A small cross was drawn on the right, $10 \mathrm{~mm}$ higher on the picture plane than the base of the standard. This was the starting point for the drawn response (see Figure 4). The position of the horizon was varied in each of the pictures and drawn at $45,65,85$, and $105 \mathrm{~mm}$ from the bottom of the picture, making Drawings A, B, C, and D. As a result of this manipulation, the ratio in which the standard was intersected by the horizon was, in order, $1 / 5,2 / 5,3 / 5$, and $4 / 5$ (lower part to whole).

To allow a very high horizon to be tested, a fifth picture (E) was included in which the configuration of standard line and cross was raised by $15 \mathrm{~mm}$. A horizon line drawn at $135-\mathrm{mm}$ elevation intersected this standard in the ratio $19 / 20$.

Design and Procedure. A 2 (subject group) $\times 5$ (horizon elevation) between-subjects design was employed. Each subject saw just one picture to avoid carry-over effects, and viewing of the picture was unrestricted as before. The subjects were seated at a table, and the picture was placed in front of them. They were tested individually and given the same instructions as in Experiment 1, except that the cross was indicated as the starting point for the response. Again, no indication was given of the identity or function of the horizon 




Figure 4. Experiment 2: The horizon was drawn at one of the five elevations shown, to give Conditions A-E. The standard line and starting cross were not moved in Conditions $A-D$ but were raised slightly in Condition $\mathrm{E}$ to maintain intersection (see text).

line. The subjects were encouraged to adjust their response with a pencil and eraser until it "looked right," and then they were asked to describe what they had done and to identify the horizontal line.

\section{Results and Discussion}

The horizon-ratio rule predicts an increase in the length of the drawn response with an increase in the elevation of the horizon. The broken line in Figure 5 is a plot of the precise response required by the horizon-ratio relation for each drawing with increasing elevation of the horizon $(50$, $75,83.3,87.5$, and $89.4 \mathrm{~mm}$ ). The bars represent the mean obtained response lengths in each condition, by artists and controls, with standard errors indicated (Overall means = $66.5,75,74.22,85.53$, and $79.2 \mathrm{~mm}$, respectively. Two control subjects produced very short responses, more than 2 standard deviations from the mean, to Drawing C. If they are excluded, as they are in Figure 5, the mean response to the drawing is $81.75 \mathrm{~mm}$.)

The figure shows that the mean responses for Drawings $\mathrm{B}, \mathrm{C}$, and D lie close to the predictions from the horizonratio relation, and they are not significantly different from them [Drawing B, $t(15)=0 ; \mathrm{C}, t(13)=1.66 ; \mathrm{D}, t(14)=$ 1.69]. It is apparent that the average response to Drawing $A$ is longer than predicted $[t(15)=4.18, p<.001]$ and to Drawing $\mathrm{E}$, somewhat shorter $[t(14)=2.76, p<.05]$. In general, however, there were many very accurate responses and especially high agreement among responders to drawings with higher horizons.

Some of the variance in response length must be due to drawing skill and the unwillingness of most subjects to correct a line once it has been drawn. Observation of subjects in this and other experiments suggests that the present paper-and-pencil technique is quite vulnerable to this source of inaccuracy. There is no objective measure of this source of variance, but it is not needed here. Responses within $7 \mathrm{~mm}$ of the predicted length are taken to be accurate ( $10 \%$ of the overall average response length) for the purposes of comparing accuracy in the different conditions. By this criterion $66.67 \%$ of responses to Drawing B were "accurate," $64.29 \%$ to Drawing C, $93.33 \%$ to Draw- ing $\mathrm{D}$, and $60 \%$ to Drawing E. More varied responses are evident in the presence of a lower horizon: only $18.75 \%$ of responses to Drawing A were accurate.

The relationship between the obtained response lengths and the predicted response lengths will be linear if responses precisely match the predictions from the horizonratio relation, and the regression line of response length on the predicted values will have slope 1 and $y$-intercept 0 . The obtained regression line has slope .374 and intercept 47.314 , again reflecting poorer performance when the horizon is either low or very high. The coefficient of determination $\left(r^{2}\right)$ indicates that only $13.1 \%$ of the variability in response length is accounted for by the horizon-ratio relation, which is, nevertheless, a significant predictor $[F(1,74)=11.169, p<.01]$. Separate analyses of the data from the trained subjects (artists) and the control group indicates that the horizon-ratio relation is a better predictor of performance for the former than for the latter [artists, $r^{2}=.35, F(1,39)=21.02, p<.0001 ;$ controls, $r^{2}=.022$, $F(1,33)=.748$, n.s.]. The slope of the regression line is significantly closer to one for the artists' data than that for the controls', because there were more accurate responses in the former group [artists' slope $=0.5$, controls' slope $=$ $0.197, t(72)=5.189, p<.001]$.

Interestingly, several subjects reported that the lowest and highest horizon lines (Drawings $A$ and $E$ ) appeared, in fact, to mark the limit of a finite surface such as a table or a floor. Fewer subjects identified the line as the horizon in these two conditions $(66.67 \%$ and $53.33 \%$, respectively, compared with $93.33 \%$ with the line in its next-to-highest position). In addition, there is an association between identification of the line as the horizon and the number of accurate responses obtained $\left[\chi^{2}(1, N=76)=19.174, p<\right.$ $.0001]$. So the number of accurate responses obtained was contingent upon the observers' identification of the line as

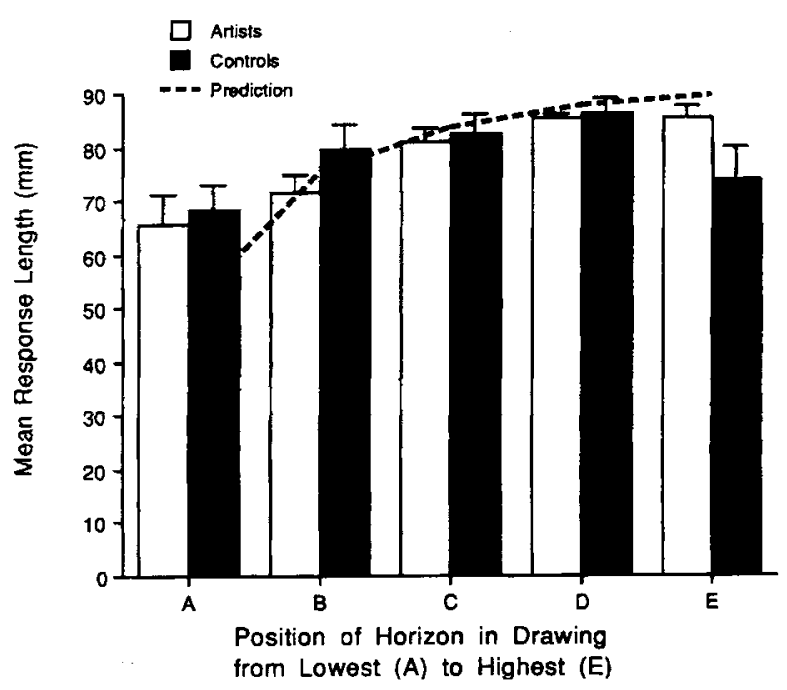

Figure 5. Experiment 2: Mean obtained response lengths, for artists and controls, in each of five conditions. The broken line indicates response lengths predicted by the horizon-ratio relation. Vertical bars indicate standard error of the mean. 
the horizon, and identification in turn depended on the line's location in the picture.

The pictures used in these experiments were extremely spare in content and so rather unusual. A horizontal line across a picture may of course represent any number of things. Perhaps the question of the identity of the line arises only in these extremely simple pictures. Limits on the effectiveness of the horizon-ratio relation in pictures may then be confined to this unusual class of pictures (and it is certainly confined to pictures and has no relevance to the perception of real scenes, in which the horizon is an optical invariant, always at eye level). Nevertheless, the association between identification of the line as the horizon and accuracy of relative size judgment certainly implies that such judgments were indeed governed by a horizonbased informative structure.

The question arises, then, how well does the horizonratio relation predict the performance of those (53) subjects who identified the line as the horizon? When their data only are regressed on the predicted response lengths, the slope is much steeper, .634, the $y$-intercept is 27.823 , and more than half the variability in response length is explained by the horizon-ratio relation $\left(r^{2}=.515\right)$. This is quite impressive, but it is still a lower level of success than that obtained in Experiment 1. An important difference between the experiments is that pencil responses are less adjustable than those made with arrow keys on a keyboard. In the latter method, subjects are more likely to discover the best response while trying out different lengths. In addition, there was opportunity for improvement with subsequent trials, whereas here, only one response was obtained from each subject.

In summary, the experiment provides further evidence that the pictured horizon can provide an adequate basis for relative size judgments. A high proportion of accurate responses was obtained, making the horizon-ratio relation a significant predictor of perceived relative size, but it is also apparent that the location of the horizon in the picture plane affected the number of accurate responses obtained. This number was highest when the horizon was near to the two thirds level, and lowest when the horizon was low in the picture.

\section{EXPERIMENT 3}

In order to argue that the perception of real size in the preceding experiments is based on the horizon-ratio relation, it might seem that a control experiment presenting similar configurations of standard line and starting point but without a horizon line is needed. In the absence of a horizon, it might be expected that drawn response lengths would be unsystematic and largely unpredictable, thus demonstrating the effectiveness of the horizon-ratio relation when the horizon is actually present. Rogers and Costall (1983), however, in their earlier experiment, observed some regularity among different subjects' responses to such a blank-field condition. There are two alternative explanations. The finding, and possibly, therefore, other findings obtained when the horizon was included in the picture, is an uninteresting artifact; perhaps there is a tendency among participants to draw the comparison just so much smaller or larger than the standard, regardless of the presence of a horizon. More interestingly, subjects may impose or exploit an implicit eye level in the picture, perhaps based on their own actual eye position, on other information in the picture, or on some assumption about the content of the picture.

A suitable test of these two alternatives requires sufficient variety in the position of the starting point relative to that of the standard line in order to rule out artifactual responses and a repeated measures design. If subjects are indeed imposing an "eye-level" or implicit horizon on the picture, only multiple observations will reveal it. The experiment is therefore a variation on Experiment 1 . As in Experiment 1, if an (in this case assumed) horizon is present and responses are dependent on it, there should be a systematic linear decrease in the length of the drawn response as the starting point approaches the horizon. When the starting point appears above the subject's assumed horizon, no response is predictable, and we may well see unsystematic responses, or at least a marked change in the pattern of responding. The exact location of the assumed horizon may vary between subjects, and it may also vary with the elevation of the standard in the picture. It is reasonable to expect, however, that it will be stable within any individual's set of responses.

\section{Method}

Subjects. There were 30 subjects, 13 "artists" (as above) and 17 control subjects with only average experience with pictures who were students at Imperial College, London. None of the subjects took part in Experiment I or 2.

Design, Procedure, and pictorial displays. The picture dimensions, apparatus, and procedure were identical to those of Experiment 1 , except that the horizon was omitted. The same instructions were given. Observers had no difficulty in imagining a three-dimensional scene, despite the extremely minimal displays. Three elevations of the standard were included: 38,76 , and $114 \mathrm{~mm}$ from the bottom edge, with 10 subjects randomly assigned to each condition. The within-subjects factor was the elevation of the starting point, which began $19 \mathrm{~mm}$ from the bottom edge of the picture and progressed upward in 30 steps of almost $6 \mathrm{~mm}$ each, ending just below the top of the picture. To provide some variety in the displays from trial to trial, the distance of the starting point from the left border of the picture was selected at random on each trial by the computer from three possible positions: 38,115 , and $153 \mathrm{~mm}$ in from the left. Thus we had a 2 (subject group) $\times 3$ (elevation of standard) $\times$ 30 (elevation of starting point) mixed within-subjects design.

\section{Results and Discussion}

All 30 subjects exhibited the predicted pattern of response to some degree. Typically, the responses decreased regularly from trial to trial until a minimum was reached (often just a one-pixel dot), and usually this was well before the end of all 30 trials. When the next trial began, observers invariably laughed with surprise. One subject commented, "Surely that's above the thing [sic] now. How confusing. I'm not sure what I'm meant to be doing now. It doesn't work." Some explained that the starting point 
was above their horizon, although no mention had been made of the horizon in introducing the experiment or in giving instructions. In all cases in which the minimum was reached before the end of the experiment, a marked change in the pattern of responding occurred. Some subjects stopped responding (saying it was impossible), some apparently shifted their assumed horizon and began a second series of decreasing responses, and some responded more randomly or produced uniformly short responses after reaching an obvious minimum response. Figure 6 shows an example of this third pattern (Subject M, an artist).

This subject's standard was at $76 \mathrm{~mm}$. The minimum response length occurred when the starting point was at $99 \mathrm{~mm}$ and presumably lay on or very close to his assumed horizon. Aggregating responses by subject group and stimulus condition preserves the pattern remarkably well, indicating a high degree of consistency between subjects. Figure 7 is a plot of the changing mean response length with increasing elevation of the starting point for artists and controls in each of the three standard elevation conditions (panels $\mathrm{a}, \mathrm{b}$, and $\mathrm{c}$ ).

Notice particularly that the point at which the minimum response length occurs varies between conditions: it is low in the picture when the standard is low, and high when the standard is high. In addition, responses are generally much shorter in the lowest standard condition and, although the patterns of responses produced by the artists and control subjects are highly similar, the artists show a more rapid decrease in response length across trials in the $38-\mathrm{mm}$ and 76-mm standard-elevation conditions than do the controls.

Analysis of the results was conducted in two stages: first, individual regression analyses were conducted on the part of each subject's data obtained before the minimum response was reached. The minimum response length for each subject was obtained by inspecting the numerical values of all responses (number of pixels drawn). In most cases, there was one response that was smaller than all preceding responses and was followed immediately by longer responses, or no further response (see Figure 6 for an example). If a second minimum occurred, the first was used. In some subjects' data, there was no obvious minimum

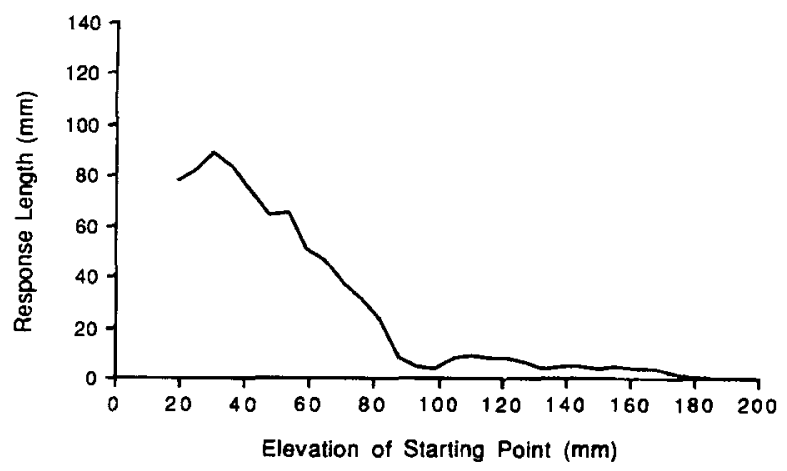

Figure 6. Experiment 3: A single subject's responses are plotted as a function of increasing elevation of the starting point. Notice the steady decrease in length to a minimum near $100-\mathrm{mm}$ elevation and then the steady short responses.
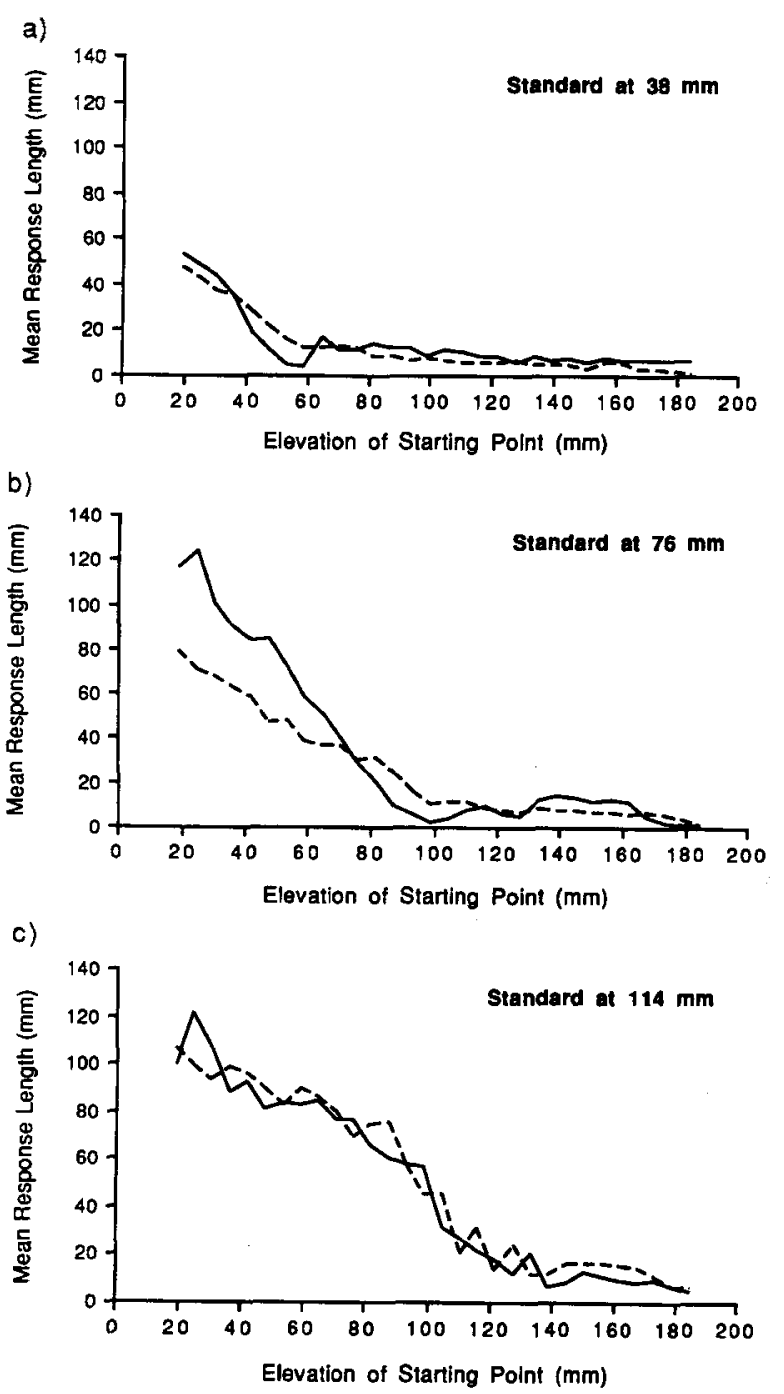

Figure 7. Experiment 3: Mean response length as a function of increasing elevation of the starting point in each of three conditions: (a) standard low at $38 \mathrm{~mm}$, (b) standard at $76 \mathrm{~mm}$, and (c) standard high at $114 \mathrm{~mm}$. The solid line indicates the artists' responses, and the broken line indicates those of the controls.

and the whole set was used in the regression analysis. The obtained individual $r^{2} \mathrm{~s}$ and $x$-intercepts ( $y$-intercept/ slope) were then used as dependent measures in two subsequent 2 (subject group) $\times 3$ (stimulus condition) analyses of variance. The $x$-intercept of the regression line conveniently indicates the position of the horizon that best supports that subject's responses. The coefficient of determination, $r^{2}$, is an indicator of the precision with which subjects maintained a linear decrease in response length with increasing elevation of the starting point.

All 30 regression analyses were highly significant. $F$ ratios ranged from 25.485 to $804.934(p<.001$ in all cases). Response curves were strongly linear up to the point of the minimum response, as predicted. Table 2 gives the mean $r^{2}$ and mean horizon position ( $x$-intercept), with standard errors, for the artists and control subjects in each 
Table 2

Means of Individual Regression Analyses for Experiment 3 (No Horizon) for Artists and Controls for Each of Three Conditions (Elevations of Standard)

\begin{tabular}{lllll}
\hline Condition & $r^{2}$ & $S E$ & Horizon* & $S E$ \\
\hline & & Artists & & \\
$38 \mathrm{~mm}$ & .909 & .055 & 61.86 & 5.614 \\
$76 \mathrm{~mm}$ & .929 & .036 & 97.788 & 1.479 \\
$114 \mathrm{~mm}$ & .851 & .041 & 162.478 & 7.197 \\
& & Controls & \\
$38 \mathrm{~mm}$ & .885 & .033 & 109.353 & 23.075 \\
$76 \mathrm{~mm}$ & .9 & .045 & 131.337 & 13.933 \\
$114 \mathrm{~mm}$ & .766 & .051 & 171.666 & 25.633 \\
\hline
\end{tabular}

*Horizon $=y$-intercept/slope

of the three stimulus conditions. The table shows an increase in the elevation of the horizon across conditions. The ANOVA revealed a significant difference among these means $[F(2,24)=11.06, p<.001]$, and trend analysis confirmed that the increase was linear $[F(1)=21.444$, $p<.0001]$. The apparent difference between artists and controls in the position of the horizon was also just significant $[F(1,24)=4.355, p<.05]$. The interaction between subject group and stimulus condition was not significant $[F(2,24)=.609]$.

The position of the average horizon, although changing in relation to the picture frame between conditions, is remarkably consistent relative to the standard. For the control subjects, it lay about a standard length above the top of the standard itself, and we can deduce that the standard was typically seen as approximately half the height of the point of observation. In the high-standard condition, the artists also positioned their assumed horizon similarly, but in the remaining two conditions, it intersected the standard just below its top. Figure 8 depicts graphically each stimulus condition and the position of the mean horizon for each subject group. The shaded areas indicate the standard error of the mean for each horizon.

From the $r^{2} \mathrm{~s}$ in Table 2, it can be seen that a very high proportion of the variability in subjects' responses is accounted for by the linear regression equation. The majority of subjects made very precise adjustments to the length of the response from trial to trial, in keeping with the suggestion that they assumed a horizon in a particular position in the picture plane which then supported their judgments of the size of the comparison. The ANOVA of the $r^{2}$ data revealed no significant differences between subject groups $[F(1,24)=1.593]$ or stimulus conditions $[F(2,24)=3.305]$ and no interaction between the two factors $[F(2,24)=.297]$.

In summary, responses in this extremely spare, blankfield experiment were remarkably ruly. There is evidence of a systematic relationship, concordant with the horizonratio relation, between the length of the drawn response and the elevation of the starting point in the picture plane. It is suggested that a horizon is assumed to be present in the picture even when not visible and not recoverable from other pictorial structures. One possibility is that this "horizon" simply reflects the observer's own eye level.
The change in the position of the apparent horizon with varying stimulus conditions (Figure 8) hints otherwise, but perhaps observers (who were free to move their heads) simply adjusted their position in front of the monitor so that the one depicted object was comfortably in front of their eyes. Further research in which the observers' eye level is directly manipulated is warranted. A second possibility is that "eye level" is imposed on the field of objects according to the known or imagined size of the depicted objects. In the present pictures, of course, absolute size was unknown. Subjects were told that the given object was a stick or a pole, and Figure 8 shows that it tended to be judged as half eye height by the controls and usually a little taller than eye height by the artists. Further research with pictures that include more information about the identity and intended size of depicted objects may well show that, in this case, observers are able to impose a "horizon" even more precisely than they did here.

\section{a) Artists}



b) Controls

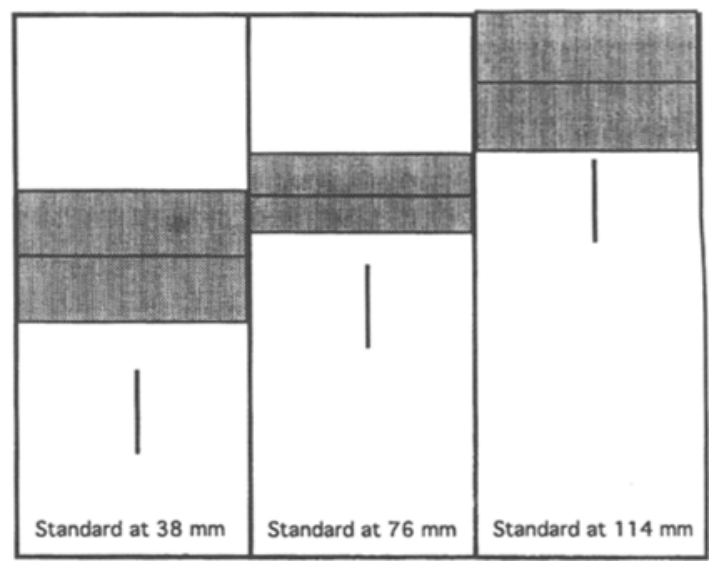

Figure 8. Experiment 3: A graphical representation of the position of the assumed horizon in each of the three standard conditions for (a) artists and (b) controls. The horizon is the mean $x$-intercept ( $y$-intercept/slope) calculated from individual regression equations. The shaded areas represent the standard error of the mean. 


\section{GENERAL DISCUSSION}

All three of the present experiments offer supporting evidence that the horizon-ratio relation provides information for the perception of relative size in pictures. This information is based on an explicit pictorial horizon when one is present. It can also be based on an implicit horizon, possibly associated with the observer's own eye level, or with the known or assumed content of the picture.

However, it is apparent from the results of the experiments reported here that there are some limits to the effectiveness of the information. First, subjects did not make use of the information when it would have resulted in too great a difference in the pictorial size of the two "same real size" objects (Experiment 1). Similar intolerance for strong perspective convergence in ordinary picture viewing has been reported before in the literature (Hagen \& Jones, 1978; Nicholls \& Kennedy, 1993; Rogers, 1995). Second, it seems that a visible horizon line must be identified as such for its informative potential to be realized. In simple pictures, such as the ones used here, the location of the line in the picture matters in this identification: if too low or too high, it will likely be considered a table edge or the limit of some other finite surface, and it will have no effect on size judgments. This restriction might not be a problem in more complex pictures, wherein other geometric structures or picture content could prevent alternative construals of the line.

In the remainder of this discussion, three arguments will be made: (1) The horizon-ratio relation is the source of information used in performance of the experimental task. Success is not due to the operation of more traditional cues. (2) The information provided by the horizonratio relation is genuinely perceptual, and success was not due to a nonperceptual, cognitive, process. (3) This pictorial structure provides information of the same sort as that available in the optic arrays from real environments, and thus no special processes are required to perceive pictures.

First, information based on the horizon-ratio relation is the most likely explanation for the results of the present study. The findings cannot be explained adequately in terms of traditional cue theory. Although the simple cues of relative size and height in the picture allow for figures to decrease in size as they become higher in the picture plane, they cannot account for the very fine adjustments to the comparison that many subjects were able to make. The pictorial size of the comparison in relation to the standard indicates only that the comparison is at some unspecified distance from the standard. The cue of relative size would prompt subjects to draw the comparison line smaller if it were farther and taller if it were nearer: How much smaller or taller is not specified by the cues. These cues alone or together are unable to predict the ruliness of behavior observed here.

Sedgwick (1973) has suggested that the cue of height in the picture might better be described as a rather simple horizon relation, if the horizon is implicit in the elevation rule. In other words, the nearer an object is to the horizon, the higher it is in the picture plane, and the farther it will be from the observer. This notion necessarily implies a limit to the ground plane and thus a limit to the height-inthe-picture rule (it does not, of course, specify where that limit lies). After a certain point, greater height in the picture plane would not indicate greater distance (as the cue would predict), because the figures would no longer appear to be on the ground. In the context of Experiment 3, this rule would predict a change in the pattern of response once the limit was reached. Such a change was evident in the results of Experiment 3, implicating a horizon relation in the task. Sedgwick (1973) has suggested that a better name for the height in the picture cue is the "ordinal distance" horizon relation. However, even in this clearer expression, the cue cannot specify how far away or how tall an object is. Because the horizon-ratio relation specifies the exact size of vertical objects resting on the ground plane, and because present results match its predictions closely in many conditions, even in the absence of a visible horizon, it is a likely explanation for the present results.

Second, evidence that the horizon-ratio relation provided useful perceptual information here can be found in the relative performance of the artists and the control subjects. The lack of important differences between the two groups indicates that the required adjustment task is truly perceptual and that success does not depend on considerable experience in producing pictures or explicit knowledge of pictorial structures or of construction rules. Furthermore, sensitivity to the horizon-ratio relation appears to be fully developed in adults and does not require unusual exposure to perspective pictures. That said, however, it is also true that a number of participants in all three experiments were astonishingly skilled in matching the comparison to the standard, and that these individuals were usually artists. The implication is that sensitivity to the horizon-ratio relation is acquired and that training or extra experience with pictures can hone sensitivity to a truly high level. Whether this skill is in fact due to the education of observers' attention to this rather subtle source of information (E. J. Gibson \& J. J. Gibson, 1955) or is instead due to an explicit understanding of the rule cannot be determined on the basis of these experiments. However, it should be noted that none of the participants were able to articulate the horizon-ratio rule even after completing the experiment. Most reported simply that they made an adjustment until the picture "looked right" or "felt right." (Further studies of the development of sensitivity to the horizonratio relation, in children and adolescents, are under way in this laboratory.)

Finally, although the horizon-ratio relation certainly seems to have been the source of useful information in the present study, the existence of conditions that constrained the effectiveness of the horizon-ratio relation, in pictures, raises the question of whether the information provided by this structure is indeed of the same sort as that provided by the optic arrays from real scenes. It is tempting to conclude with J. J. Gibson (1971) that it is, and therefore that no special theory of picture perception is needed. However, the constraints, observed here, on the effectiveness of the horizon-ratio relation are restricted to pictures and 
are not relevant to the real scenes that they represent, indicating an important distinction between the two. The distinction is important, because the motivation for the present study was the question of the existence of pictorial information, which Gibson (1971) has argued is of the "same kind of information that is found in the ambient optic array of an ordinary environment" (p. 31); and Gibson has argued that picture perception is due to the same kind of process, the direct detection of informative structure (cf. J. J. Gibson, 1979). Pictures are thus subsumed under Gibson's more general theory of perception.

The results of the present study, however, suggest the need for some important qualifications to Gibson's theory of pictures. For any optical structure to be truly meaningful, certain limiting conditions (or constraints) must be met under which that structure unambiguously specifies some aspect of reality (Barwise \& Perry, 1983). The constraints may well be different when the structure is not revealed through the continuous transformations of the optic array (in the perception of real scenes) but is frozen upon a picture surface. Pictures are different from real scenes in that they have much greater potential for ambiguity, and thus pictorial structures will require the satisfaction of some additional constraints in order for them to carry the same information as do their real scene equivalents. For example, we have seen that available information may be effective only when important features are adequately identified and when the degree of perspective convergence employed is within tolerable limits (see also Hagen \& Jones, 1978; Nicholls \& Kennedy, 1993; Rogers, 1995). Future research will address the nature and role of these and other constraints on pictorial information more fully.

In conclusion, the horizon-ratio relation can be an effective source of information for size in pictures. This information is the same kind as that found in the optic array from real scenes, but it requires the satisfaction of some additional constraints if it is to be fully effective. When the constraints are met, and when the information is detected, the perception of pictorial space can proceed in the same way as does the perception of the real scenes that it depicts.

\section{REFERENCES}

Barwise, J., \& Perry, J. (1983). Situations and attitudes. Cambridge, MA: MIT Press.

BENSON, C., \& Yonas, A. (1973). Development of sensitivity to static pictorial depth information. Perception, 13, 361-366.

Bingham, G. P. (1993a). Perceiving the size of trees: Biological form and the horizon ratio. Perception \& Psychophysics, 54, 485-495.

Bingham, G. P. (1993b). Perceiving the size of trees: Form as information about scale. Joumal of Experimental Psychology: Human Perception \& Performance, 19, 1139-1161.

Gibson, E. J., \& GiBson, J. J. (1955). Perceptual learning: Differentiation or enrichment? Psychological Review, 62, 32-41.

Gibson, J. J. (1966). The senses considered as perceptual systems. Boston: Houghton Mifflin.

GIBSON, J. J. (1971). The information available in pictures. Leonardo, 4 , 27-35.

GiBson, J. J. (1979). The ecological approach to visual perception. Boston: Houghton Mifflin.
Granrud, C. E., Yonas, A., \& Opland, E. A. (1985). Infants' sensitivity to the depth cue of shading. Perception \& Psychophysics, 37, 415-419.

HAGEN, M. A. (1980). The perception of pictures (Vols. 1-2). New York: Academic Press.

HAGEN, M. A., \& Jones, R. K. (1978). Differential patterns of preference for modified linear perspective in children and adults. Journal of Experimental Child Psychology, 26, 205-215.

IrESoN, J., \& MCGURK, H. (1985). Utilization of static and kinetic information for depth by young Malawians. Journal of Experimental Child Psychology, 40, 233-243.

JAHODA, G., \& MCGuRK, H. (1974). Pictorial depth perception: A developmental study. British Journal of Psychology, 65, 141-148.

MARK, L. S. (1987). Eyeheight-scaled information about affordances: A study of sitting and stair-climbing. Journal of Experimental Psychology: Human Perception \& Performance, 13, 361-370.

Nicholls, A. L., \& Kennedy, J. M. (1993). Angular subtense effects on perception of polar and parallel projections of cubes. Perception \& Psychophysics, 54, 763-772.

OLSON, R. K. (1975). Children's sensitivity to pictorial depth information. Perception \& Psychophysics, 17, 59-64.

Olson, R. K., \& Boswell, S. (1976). Pictorial depth sensitivity in twoyear-old children. Child Development, 47, 1175-1178.

RoGERS, S. (1995). Perceiving pictorial space. In W. Epstein and S. Rogers (Eds.) Handbook of perception and cognition: Vol. 5. Perception of space and motion (2nd ed., pp. 119-163). San Diego, CA: Academic Press.

Rogers, S., \& Costall, A. (1983). On the horizon: Picture perception and Gibson's concept of information. Leonardo, 16, 180-182.

SEDGwICK, H. A. (1973). The visible horizon: A potential source of visual information for the perception of size and distance. Dissertation Abstracts International, 34, 1301B-1302B. (University Microfilms No. 73-22530)

SEDGWICK, H. A. (1980). The geometry of spatial layout in pictorial representation. In M. A. Hagen, The perception of pictures (Vol. 1, pp. 33-90). New York: Academic Press.

WARREN, W. H. (1984). Perceiving affordances: Visual guidance of stairclimbing. Journal of Experimental Psychology: Human Perception \& Performance, 10, 683-703.

WARREN, W. H., \& WHANG, S. (1987). Visual guidance of walking through apertures: Body-scaled information for affordances. Journal of Experimental Psychology: Human Perception \& Performance, 13, 371-383.

WiLcox, B. L., \& TEghtsoonian, M. (1971). The control of relative size by pictorial depth cues in children and adults. Journal of Experimental Child Psychology, 11, 413-429.

YONAS, A., \& Hagen, M. (1973). Effects of static and kinetic depth information on the perception of size in children and adults. Journal of Experimental Child Psychology, 15, 254-265.

\section{NOTES}

1 . Note that the observation that relative size may be specified independently of distance marks an important deviation from the existing assumption of a close link between the two.

2. J. J. Gibson's ecological theory of visual perception is not a psychophysical theory. It does not decree that available information must necessarily be effective in perception. It does, however, does allow specific predictions to be made. The horizon-ratio relation specifies the height of one depicted object precisely in relation to that of another, so that observers who utilize this source of information should produce very accurate responses. Those who do not utilize the structure may well produce aberrant responses. The aggregate performance of the group could therefore reflect responses based on a wide variety of strategies. The relevant prediction does not concern this aggregate performance but rather the existence of some very accurate responders within the group.

(Manuscript received March 15, 1995; revision accepted for publication June 12, 1995.) 